\title{
Osteoarthrosis of Temporomandibular Joint Related to the Defects of Posterior Dentition: A Retrospective Study
}

\author{
Jitka Levorová, Vladimír Machoň, Anasuya Guha, René Foltán \\ Department of Oral and Maxillofacial Surgery, Department of Dental Medicine, \\ First Faculty of Medicine, Charles University and General University Hospital \\ in Prague, Prague, Czech Republic
}

Received June 7, 2016; Accepted November 21, 2016.

Key words: Temporomandibular joint - Osteoarthrosis/osteoarthritis Posterior dentition - Supporting zones - OA etiologic factors

\begin{abstract}
Osteoarthrosis (OA) of temporomandibular joint (TMJ) is a progressive degenerative disease, gradually affecting cartilage, synovial membrane and bone structures. OA of TMJ clinically manifests with joint noises, pain and restricted mouth opening. In late stages, it results in severe damage of TMJ structures and development of ankylosis. Osteoarthrosis is a multifactorial disease; the occurrence is associated with TM] overloading. The cohort included 619 patients [538 women (87\%) and 81 men (13\%), with average age 40.6 years (age range $8-89$ years)] with TMJ disorder, who were examined in the year 2014 in Department of Dental Medicine, First Faculty of Medicine, Charles University and General University Hospital in Prague, Czech Republic. The aim of this retrospective study was to analyse, if the lack of posterior teeth (supporting teeth zones) is the main etiologic factor of osteoarthrosis of TMJ. Diagnosis of OA was established on the clinical signs and the panoramic $X$-ray signs. Simultaneously other etiologic factors of $O A$ were assessed. The presence of OA changes on X-ray had 171 patients (i.e. 27.6\% of the total number of 619). $17.5 \%$ from these patients with OA had defect in posterior dentition. Other aetiological factors (stress, skeletal or vertebrogenous disorders) showed higher incidence of OA changes on X-ray. Defect of posterior dentition is not negligible, but it is not the main aetiological factor for osteoarthrosis of TMJ.
\end{abstract}

Mailing Address: MDDr. Jitka Levorová, Department of Dental Medicine, First Faculty of Medicine, Charles University and General University Hospital in Prague, U Nemocnice 2, 12000 Prague 2, Czech Republic; Mobile Phone: +420 728022 635; e-mail: Jitka.levorova@gmail.com 


\section{Introduction}

Temporomandibular joint (TMJ) osteoarthrosis (OA) is a chronic disease, which manifests with progressive degenerative changes of joint structures. OA develops because of imbalance between reparative and degenerative processes of the joint (Mercuri, 2008; Adebajo, 2009; Machoň and Hirjak, 2014).

TMJ OA might not be clinically manifested and might be accidentally found on panoramic radiograph during the initial phase (Figure 1). The first clinical symptoms include sound phenomena associated with jaw movements (crepitus or clicking in case of articular disc derangement or perforation). With the progression of degenerative changes, pain and restriction of mouth opening usually occur. In the

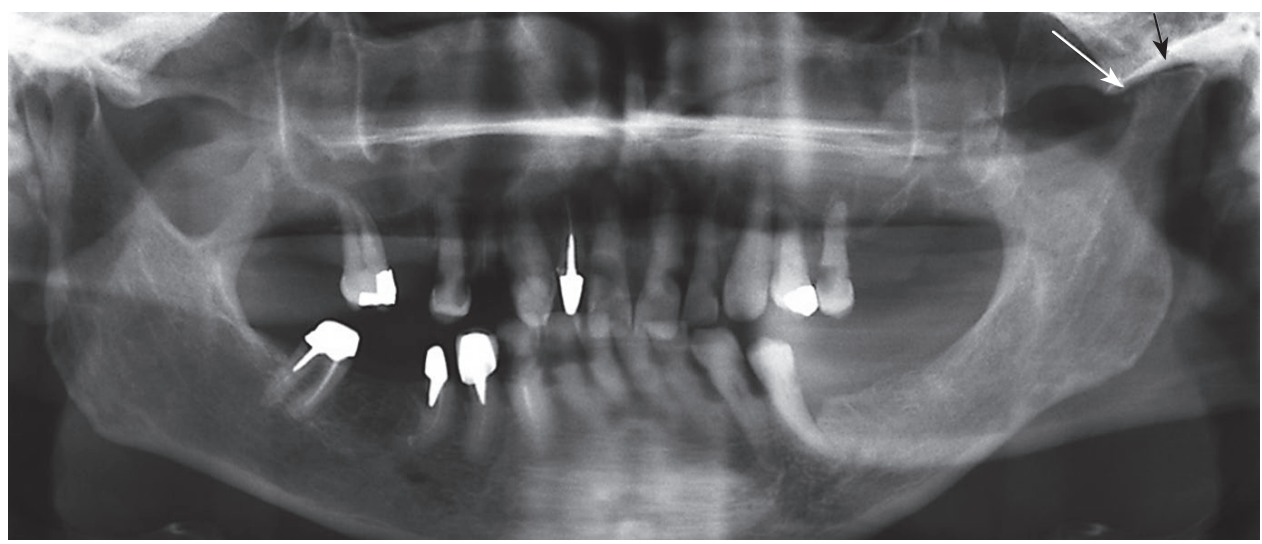

Figure 1 - Panoramic radiograph shows flattening of left condylar head of mandible, narrowing of joint space; incongruence of head surface and loose body in anterior aspect: degenerative changes.

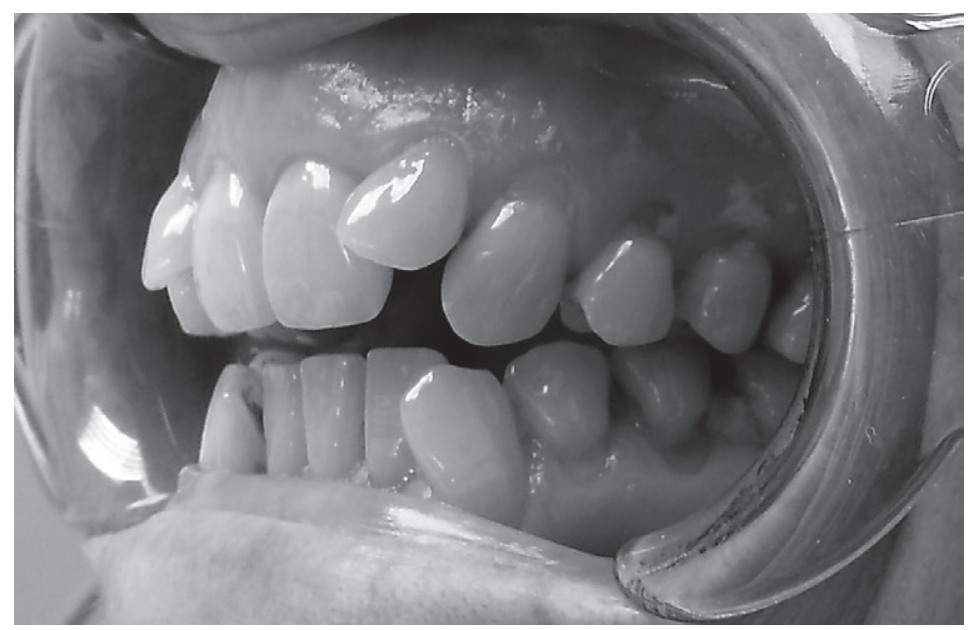

Figure 2 - Angle Class II malocclusion and anterior open bite, which developed as a result of severe bilateral osteoarthrosis of temporomandibular joint. 


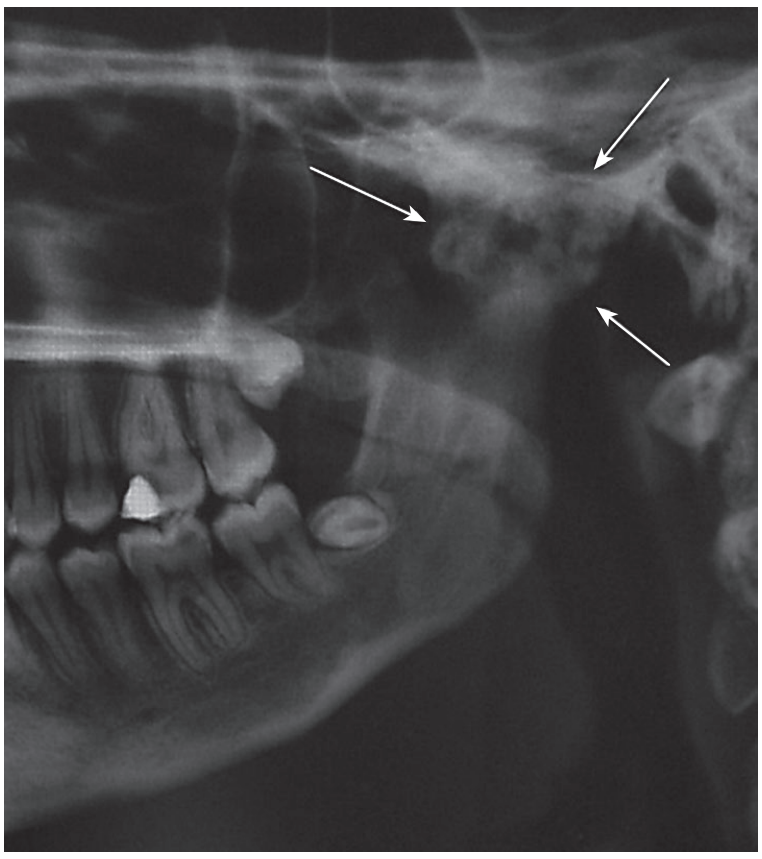

Figure 3 - Bone mass representing ankylosis of left temporomandibular joint in young patient: result of a trauma.

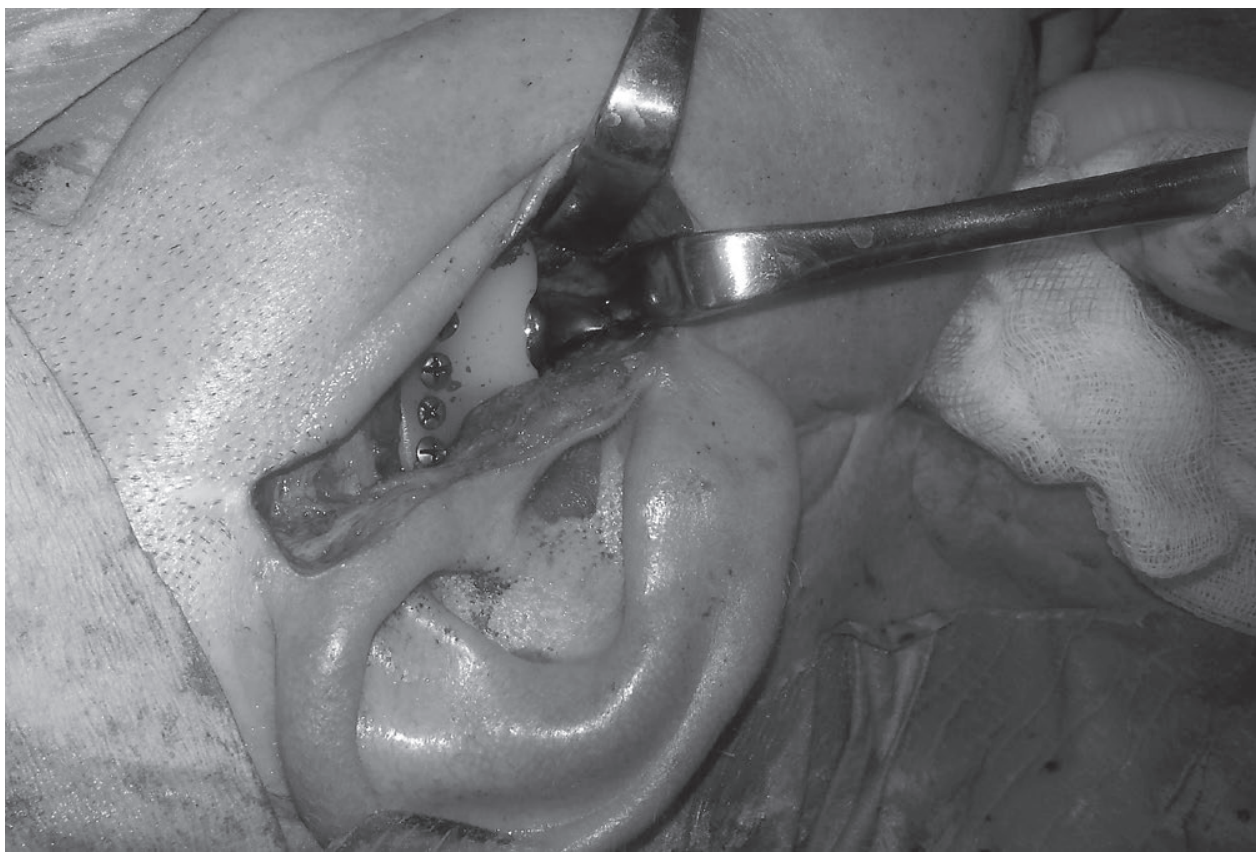

Figure 4 - Intraoperative photo of temporomandibular joint total alloplastic prosthesis (TMJ Concepts, Ventura, California, USA). 
late stages of the disease, the height of the condylar process may be shortened. This condition leads to anterior open bite and development of Angle Class II (Figure 2). Degenerative changes of joint surfaces and disc perforation are risk factors for the development of ankylosis (pathological fusion between mandibular condylar head and glenoid fossa) (Figure 3). The most extensive arthritic changes and ankylosis are an indication for total joint replacement (Figure 4); other treatment modalities for OA are conservative, miniinvasive (arthrocenthesis, arthroscopy) or other types of open surgery, based on the severity of degenerative changes (Mercuri, 2008; Adebajo, 2009; Machoň and Hirjak, 2014).

Pathogenesis of OA lays in increased stress to joint structures that leads to an increase in intra-articular pressure and continuous disturbance of blood supply of the retrodiscal tissue. Cycle of transient hypoxia followed by reoxygenation of the retrodiscal tissue provokes the release of free oxygen radicals, which inhibit the synthesis of hyaluronic acid (HA) in the synovial joint. Interleukins and prostaglandins are released into joint spaces and induce activation of proteolytic enzymes (matrix metalloproteinases), which take part on chondromalacia development. Simultaneously occurring changes in composition of synovial fluid, including the lack of HA, lead to the loss of nutritional efficiency and lubricating function of the fluid. This condition results in increased friction and subsequently arthritic changes of subchondral bone (Blake et al., 1989; Grootveld et al., 1991; Nitzan, 1994, 2001; Laskin et al., 2006; Okeson, 2007; Mercuri, 2008; Machoň and Hirjak, 2014).

Increased stress may be caused by extensive load (macrotrauma or chronic microtraumatization as bruxism or functional overload). When this occurs in normal joint structures, the disease is called primary OA. Abnormal or even normal load acting against compromised joint structures (congenital collagen defects; metabolic, rheumatoid, infectious diseases) plays a role in the development of secondary OA (Laskin et al., 2006; Okeson, 2007; Mercuri, 2008; Machoň and Hirjak, 2014).

Unstable occlusion (bite/skeletal disorders, premature teeth contact) is one of the aetiological factors. Influence of orthopaedic instability (breakdown in relations between maximum intercuspidation and musculoskeletal stable position) on joint structures is a theoretical matter (Pullinger et al., 1993; Pullinger and Seligman, 2000). In the real situation, unstable occlusion increases the activity of masticatory muscles and joint surfaces are loaded during the mandibular movements. Relationship to the lateral part of the teeth is as following: when the maximum intercuspation of premolars and molars on both sides is achieved, there is a distribution of masticatory forces on all these teeth and load of the joint structures is minimized. If one/more teeth from this relationship are detached, this increases the force acting on the remaining teeth involved in that intercuspation and, the force transferred to the structure of the TMJ increases. Analogously, when only teeth on one side are in contact, then the masticatory forces transfer to 
contralateral side. When there is a complete lack of posterior teeth (from the first premolar to the second or third molar), contact of the anterior teeth (in range between canines) is achieved, then it is not a stable occlusion (Okeson, 2007).

The authors focused on a group of patients with the lack of support zones (distal teeth) to confirm or reject the hypothesis, that a one- or two-sided lack of support zones causes overload of TMJ and thus contributes to the formation of degenerative changes in the structures of the temporomandibular joint (Pullinger et al., 1993; Pullinger and Seligman, 2000; Okeson, 2007).

\section{Material and Methods}

The study includes patients, who were examined in the Department of Dental Medicine, First Faculty of Medicine, Charles University and General University Hospital in Prague, Czech Republic in the year 2014. From a total of 1,024 patients with temporomandibular joint disorders, patients with osteological, autoimmune, metabolic and rheumatoid diseases had been excluded.

After exclusion, the study consisted of 619 individuals. Patients were aged 8-89 years; the average age was 40.6 years. Women accounted for $87 \%$ of all patients (538 patients); the remaining $13 \%$ were men ( 81 patients). Osteoarthrosis was evaluated based on the presence of degenerative changes on the panoramic radiograph; therefore, the study included both symptomatic and non-symptomatic patents with OA.To evaluate the defects of the support zones, panoramic radiographs and clinical examination were used. A defect of the support zone was defined as one- or two-sided shortening of the upper or lower or both dental arches (from first premolar to second or third molar). Patients were evaluated for other possible etiologic factors: significant psychical stress load, skeletal defects, vertebral disorders (pain in the cervical spine and neck or neck muscles), bruxism, trauma (fractures of the lower jaw in the personal history).

\section{Results}

The examined group consisted of 619 patients (TMJ disorder, without further osteological, autoimmune, rheumatoid, metabolic diseases).

538 women (87\%) and 81 men (13\%).

The average age was 40.6 years (age range $8-89$ years).

The presence of OA changes on X-ray:

171 patients (i.e. $27.6 \%$ of the total number of 619 ).

These were 162 women (94.8\%) and 9 men (5.2\%).

The average age was 46.3 years (age range 33-89 years).

Defect of supporting zones in patients with OA changes on X-ray:

Defect of supporting zones was found in 30 patients (17.5\%) out of 171 patients with OA. 
These were 9 men (30\%) and 21 women (70\%).

The average age was 57.9 years (age range $35-83$ years).

When men only were evaluated with OA ( 9 patients), then the absence of the support zones was in all patients $(100 \%)$.

If only women were evaluated with OA (162 patients), then the absence of the support zones was only 21 patients (12.9\%).

Other etiological factors in patients with OA changes on X-ray (171 patients) (Figure 5):

$31 \%$ (51 patients) - significant stress load

$30 \%$ (50 patients) - skeletal malformation (hypoplastic mandible, Angle Class II)

$28 \%$ (46 patients) - vertebrogenous disorders

$12 \%$ (20 patients) - bruxism

$3 \%$ (5 patients) - trauma (fractures of the mandible)

\section{Discussion}

Prevalence of osteoarthrosis, as well as overall temporomandibular joint diseases, is higher in women (70-72\% of cases) (Pullinger et al., 1993; Pullinger and Seligman, 2000). The same result was found in this study (females $87 \%$, males $13 \%$ with TMJ disorders; resp. $94.8 \%$ females and $5.2 \%$ males with OA). The aetiology of this disease is now considered as multifactorial. Increased psychological stress accompanied by anxiety and depression is reported as a vital factor (Dworkin and Massoth, 1994; Suvinen and Reade, 1995; Ferrando et al., 2004). This fact was confirmed in this study: a total of $31 \%$ of patients in this study diagnosed with OA admitted increased psychological burden. Patients often compensate increased psychological stress by conscious or unconscious parafunctional activities, which cause chronic microtraumatization of the joint and subsequent development of inflammatory degenerative changes. The mechanism of chronic TMJ overload operates in bruxism, skeletal defects (especially Angle Class II, open

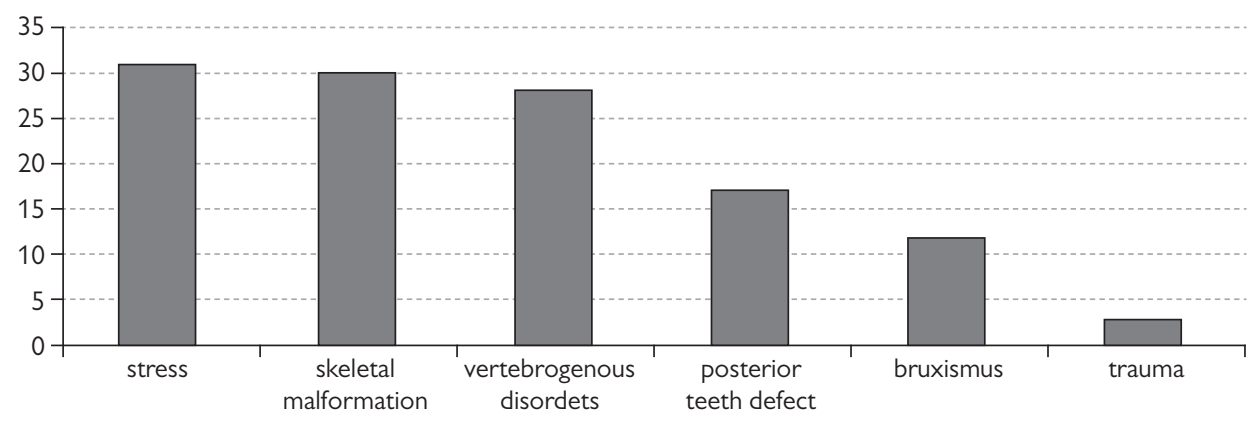

Figure 5 -Aetiological factors of osteoarthrosis. 
bite) and vertebrogenous problems (Israel and Scrivani, 2000; Güler et al., 2003). Macrotrauma as the cause of developing OA of TMJ was only a marginal factor ( $3 \%$ of the patients had a history of trauma to the mandible; a total of 5 patients with a fracture of the lower jaw in all cases). Extensive studies, Probert et al. (1994) comprising 20,763 patients after car accident, showed the presence of TMJ disorder even only in 28 patients (i.e. $0.13 \%$ ).

Pullinger et al. (1993), Pullinger and Seligman (2000) and Okeson (2007) evaluate one or both sides shortened dental arch as an important factor for overloading of ipsilateral/bilateral joint structures. In this study, degenerative changes developed only in $17.5 \%$ of patients with defect of supporting zones. The study shows, that the lack of posterior teeth is not the main aetiological factor of TMJ OA. Okeson (2007) refers to 57 other authors who diverge in opinion on relation between failure of occlusion and TMJ disorders. A total of 22 of these authors reject the relation, and other authors consider the defects of occlusion as the most important factor in the TMJ disorders development (Tsolka et al., 1994; Ciancaglini et al., 1999).

When only men with OA were evaluated (9 patients), then the defect in posterior dentition was seen in all these patients (100\%). When considering the same condition in women: 21 patients with OA had the defect of posterior dentition (i.e. $12.9 \%$ out of 162 patients with OA). This result correlates with the work of Magnusson et al. (2010), which presents the lack of support zones as $s$ significant risk factor of OA for men, not for women (per his work, increasing age is a risk factor for women is a risk factor). Data can be distorted by different gender representation in our compared to Magnusson's study (Magnusson investigates 170 men, 89 women, our study includes 81 men and 538 women). In studies of several different authors dealing with the masticatory forces (and their association with TMJ disorders), it was found, that men use larger chewing power than women (Shinogaya et al., 2001; Ferrario et al., 2004; Pizolato et al., 2007; Koc et al., 2010), with a difference of up to $300 \mathrm{~N}$ (that is $30 \mathrm{~kg} \times \mathrm{m} / \mathrm{s}^{2}$ !) (Waltimo and Könönen, 1993). Conversely, a higher value of the intra-articular pressure was measured in females than males, both at closed mouth position at rest and during clenching of the teeth, i.e. showing increased activity of the masticatory muscles (Nitzan, 2001).

\section{Conclusion}

Surprisingly, only a small percentage of patients examined for TMJ disorders in this study, had unsatisfactory dental situation in terms of lack of posterior teeth. The study shows, that lack of posterior dentition is not the essential factor in the development of osteoarthrosis. On the other hand, the percentage of patients, who have arthritic changes of TMJ and shortened dental arch at the same time, is not negligible. It indicates that these factors are involved in the development of osteoarthrosis, as well as in the development of other TMJ disorders. 


\section{References}

Adebajo, A. (2009) ABC of Rheumatology, $4^{\text {th }}$ Edition. BMJ Books, London.

Blake, D. R., Unsworth, J., Outhwaite, J. M., Morris, C. J., Merry, P., Kidd, B. L., Ballard, R., Gray, L., Lunec, J. (1989) Hypoxic-reperfusion injury in the inflamed human joint. Lancet 333(8633), 289-293.

Ciancaglini, R., Gherlone, E. F., Radaelli, G. (1999) Association between loss of occlusal support and symptoms of functional disturbances of the masticatory system. J. Oral Rehabil. 26(3), 248-253.

Dworkin, S. F., Massoth, D. L. (1994) Temporomandibular disorders and chronic pain: disease or illness? J. Prosthet. Dent. 72(1), 29-38.

Ferrando, M., Andreu, Y., Galdón, M. J., Durá, E., Poveda, R., Bagán, J.V. (2004) Psychological variables and temporomandibular disorders: distress, coping, and personality. Oral Surg. Oral Med. Oral Pathol. Oral Radiol. Endod. 98(2), 153-160.

Ferrario, V. F., Sforza, C., Serrao, G., Dellavia, C., Tartagilia, G. M. (2004) Single tooth bite forces in healthy young adults. J. Oral Rehabil. 31, 18-22.

Grootveld, M., Henderson, E. B., Farrell, A., Blake, D. R., Parkes, H. G., Haycock, P. (1991) Oxidative damage to hyaluronate and glucose in synovial fluid during exercise of the inflamed rheumatoid joint. Detection of abnormal low-molecular-mass metabolites by proton-n.m.r. spectroscopy. Biochem. J. 273(Pt 2), 459-467.

Güler, N., Yatmaz, P. I., Ataoglu, H., Emlik, D., Uckan, S. (2003) Temporomandibular internal derangement: Correlation of MRI findings with clinical symptoms of pain and joint sounds in patients with bruxing behaviour. Dentomaxillofac. Radiol. 32(5), 304-310.

Israel, H. A., Scrivani, S. J. (2000) The interdisciplinary approach to oral, facial and head pain. J. Am. Dent. Assoc. 131(7), 919-926.

Koc, D., Dogan, A., Bek, B. (2010) Bite force and influential factors on bite force measurements: a literature review. Eur. J. Dent. 4(2), 223-232.

Laskin, D. M., Greene, C. S., Hylander,W. L. (2006) Temporomandibular Disorders: An Evidence-based Approach to Diagnosis and Treatment. Quintessence Publishing, Hanover Park.

Machoň,V., Hirjak, D. (2014) Atlas Léčby Onemocnění Temporomandibulárního Kloubu. Triton, Praha.

Magnusson, C., Nilsson, M., Magnusson, T. (2010) Degenerative changes in human temporomandibular joints in relation to occlusal support. Acta Odontol. Scand. 68(5), 305-311.

Mercuri, L. G. (2008) Osteoarthritis, osteoarthrosis, and idiopathic condylar resorption. Oral Maxillofac. Surg. Clin. North Am. 20(2), 169-183.

Nitzan, D.W. (1994) Intraarticular pressure in the functioning human temporomandibular joint and its alteration by uniform elevation of the occlusal plane. J. Oral Maxillofac. Surg. 52(7), 671-679.

Nitzan, D.W. (2001) The process of lubrication impairment and its involvement in temporomandibular joint disc displacement: a theoretical concept. J. Oral Maxillofac. Surg. 59(1), 36-45.

Okeson, J. P. (2007) Management of Temporomandibular Disorders and Occlusion, $6^{\text {th }}$ Edition. Mosby Elsevier, St. Louis.

Pizolato, R. A., Gavião, M. B., Berretin-Felix, G., Sampaio, A. C., Trindade, A. S. Jr. (2007) Maximal bite force in young adults with temporomandibular disorders and bruxism. Braz. Oral Res. 21(3), 278-283.

Probert, T. C., Wiesenfeld, D., Reade, P. C. (1994) Temporomandibular pain dysfunction disorder resulting from road traffic accidents - an Australian study. Int. J. Oral Maxillofac. Surg. 23(6 Pt 1), 338-341.

Pullinger, A. G., Seligman, D. A. (2000) Quantification and validation of predictive values of occlusal variables in temporomandibular disorders using a multifactorial analysis. J. Prosthet. Dent. 83(1), 66-75.

Pullinger, A. G., Seligman, D. A., Gornbein, J. A. (1993) A multiple logistic regression analysis of the risk and relative odds of temporomandibular disorders as a function of common occlusal features. J. Dent. Res. 72(6), 968-979. 
184) Prague Medical Report / Vol. 117 (2016) No. 4, p. 176-184

Shinogaya, T., Bakke, M., Thomsen, C. E., Vilmann, A., Sodeyama, A., Matsumoto, M. (2001) Effects of ethnicity, gender and age on clenching force and load distribution. Clin. Oral Investig. 5(1), 63-68.

Suvinen, T. I., Reade, P. (1995) Temporomandibular disorders: A critical review of the nature of pain and its assessment. J. Orofac. Pain 9(4), 317-339.

Tsolka, P., Fenlon, M. R., McCullock, A. J., Preiskel, H.W. (1994) A controlled clinical, electromyographic, and kinesiographic assessment of craniomandibular disorders in women. J. Orofac. Pain 8(1), 80-89.

Waltimo, A., Könönen, M. (1993) A novel bite force recorder and maximal isometric bite force values for healthy young adults. Scand. J. Dent. Res. 101(3), 171-175. 